\title{
Homoclinic Orbits for a Class of Nonperiodic Hamiltonian Systems with Some Twisted Conditions
}

\author{
Qi Wang ${ }^{1}$ and Qingye Zhang ${ }^{2}$ \\ ${ }^{1}$ Institute of Contemporary Mathematics, School of Mathematics and Information Science, Henan University, Kaifeng 475000, China \\ ${ }^{2}$ Department of Mathematics, Jiangxi Normal University, Nanchang 330022, China
}

Correspondence should be addressed to Qi Wang; mathwq@henu.edu.cn

Received 4 January 2013; Accepted 1 April 2013

Academic Editor: Changbum Chun

Copyright ( 2013 Q. Wang and Q. Zhang. This is an open access article distributed under the Creative Commons Attribution License, which permits unrestricted use, distribution, and reproduction in any medium, provided the original work is properly cited.

By the Maslov index theory, we will study the existence and multiplicity of homoclinic orbits for a class of asymptotically linear nonperiodic Hamiltonian systems with some twisted conditions on the Hamiltonian functions.

\section{Introduction and Main Results}

Consider the following first-order nonautonomous Hamiltonian systems

$$
\dot{z}=J H_{z}(t, z),
$$

where $z: \mathbb{R} \rightarrow \mathbb{R}^{2 N}, J=\left(\begin{array}{cc}0 & -I_{N} \\ I_{N} & 0\end{array}\right), H \in C^{1}\left(\mathbb{R} \times \mathbb{R}^{2 N}, \mathbb{R}\right)$ and $\nabla_{z} H(t, z)$ denotes the gradient of $H(t, z)$ with respect to $z$. As usual we say that a nonzero solution $z(t)$ of (HS) is homoclinic (to 0 ) if $z(t) \rightarrow 0$ as $|t| \rightarrow \infty$.

As a special case of dynamical systems, Hamiltonian systems are very important in the study of gas dynamics, fluid mechanics, relativistic mechanics and nuclear physics. However it is well known that homoclinic solutions play an important role in analyzing the chaos of Hamiltonian systems. If a system has the transversely intersected homoclinic solutions, then it must be chaotic. If it has the smoothly connected homoclinic solutions, then it cannot stand the perturbation, and its perturbed system probably produces chaotic phenomena. Therefore, it is of practical importance and mathematical significance to consider the existence of homoclinic solutions of Hamiltonian systems emanating from 0 .

In the last years, the existence and multiplicity of homoclinic orbits for the first-order system (HS) were studied extensively by means of critical point theory, and many results were obtained under the assumption that $H(t, z)$ depends periodically on $t$ (see, e.g., [1-12]). Without assumptions of periodicity, the problem is quite different in nature and there is not much work done so far. To the best of our knowledge, the authors in [13] firstly obtained the existence of homoclinic orbits for a class of first-order systems without any periodicity on the Hamiltonian function. After this, there were a few papers dealing with the existence and multiplicity of homoclinic orbits for the first-order system (HS) in this situation (see, e.g., [14-17]).

In the present paper, with the Maslov index theory of homoclinic orbits introduced by Chen and Hu in [18], we will study the existence and multiplicity of homoclinic orbits for (HS) without any periodicity on the Hamiltonian function. To the best of the author's knowledge, the Maslov index theory of homoclinic orbits is the first time to be used to study the existence of homoclinic solutions. We are mainly interested in the Hamiltonian functions of the form

$$
H(t, z)=-L(t) z \cdot z+R(t, z),
$$

where $L$ is a $2 N \times 2 N$ symmetric matrix valued function. We assume that $\left(L_{1}\right) L \in C\left(\mathbb{R}, \mathbb{R}^{N^{2}}\right)$, and there are $\alpha, c>0, t_{0} \geq 0$ and a constant matrix $P$, satisfying

$$
P L(t)-c|t|^{\alpha} I_{2 N} \geq 0, \quad \forall|t| \geq t_{0},
$$

where $I_{2 N}$ is the identity map on $\mathbb{R}^{2 N}$, and for a $2 N \times 2 N$ matrix $M$, we say that $M \geq 0$ if and only if

$$
\inf _{\xi \in \mathbb{R}^{2 N},|\xi|=1} M \xi \cdot \xi \geq 0 .
$$


In $\left(L_{1}\right)$, if $P=\left(\begin{array}{cc}0 & I_{N} \\ I_{N} & 0\end{array}\right)$, then $\left(L_{1}\right)$ is similar to the condition $\left(R_{0}\right)$ in [14]. But the restrictions on $R(t, z)$ will be different from [14], and we will give some examples in Remark 5. If $P=$ $\pm I_{2 N}$ or $P=\left(\begin{array}{cc}I_{N+m} & 0 \\ 0 & -I_{N-m}\end{array}\right)$ in condition $\left(L_{1}\right)$, for example, it is quite different from the existing results as authors known. In short, condition $\left(L_{1}\right)$ means that the eigenvalues of $L(t)$ will tend to $\pm \infty$ with the speed no less than $|t|^{\alpha}$. But $\left(L_{1}\right)$ does not contain all of these cases. For examples, let $N=1$ and $L(t)=$ $|t|^{\alpha}\left(\begin{array}{cc}\cos 2 t & \sin 2 t \\ \sin 2 t & -\cos 2 t\end{array}\right)$, we have the eigenvalues of $L(t)$ are $\pm|t|^{\alpha}$, but there is no constant matrix $P$ satisfying $P L(t)-c|t|^{\alpha} I_{2 N} \geq$ 0 , for all $|t| \geq t_{0}$.

Denote by $\widetilde{F}$ the self-adjoint operator $-\mathscr{J}(d / d t)+L(t)$ on $L^{2} \equiv L^{2}\left(\mathbb{R}, \mathbb{R}^{2 N}\right)$, with domain $D(\widetilde{F})=H^{1}\left(\mathbb{R}, \mathbb{R}^{2 N}\right)$ if $L(t)$ is bounded and $D(\widetilde{F}) \subset H^{1}\left(\mathbb{R}, \mathbb{R}^{2 N}\right)$ if $L(t)$ is unbounded. Let $|\widetilde{F}|$ be the absolute value of $\widetilde{F}$, and let $|\widetilde{F}|^{1 / 2}$ be the square root of $|\widetilde{F}| . D(\widetilde{F})$ is a Hilbert space equipped with the norm

$$
\|z\|_{\widetilde{F}}=\|(I+|\widetilde{F}|) z\|_{L^{2}}, \quad \forall z \in D(\widetilde{F}) .
$$

Let $E=D\left(|\widetilde{F}|^{1 / 2}\right)$, and define on $E$ the inner product and norm by

$$
\begin{gathered}
(u, v)_{E}=\left(|\widetilde{F}|^{1 / 2} u,|\widetilde{F}|^{1 / 2} v\right)_{2}+(u, v)_{2}, \\
\|u\|_{E}=(u, u)_{E}^{1 / 2},
\end{gathered}
$$

where $(\cdot, \cdot)_{L^{2}}$ denotes the usual inner product on $L^{2}\left(\mathbb{R}, \mathbb{R}^{2 N}\right)$. Then, $E$ is a Hilbert space. It is easy to see that $E$ is continuously embedded in $H^{1 / 2}\left(\mathbb{R}, \mathbb{R}^{2 N}\right)$, and we further have the following lemma.

Lemma 1. Suppose that $L$ satisfies $\left(L_{1}\right)$. Then $E$ is compactly embedded in $L^{p}\left(\mathbb{R}, \mathbb{R}^{2 N}\right)$ with the usual norm $\|\cdot\|_{L^{p}}$ for any $1 \leq p \in(2 /(1+\alpha), \infty)$.

This lemma is similar to Lemmas 2.1-2.3 in [13], and we will prove it in Section 3. Define the quadratic form $Q$ on $E$ by

$$
Q(u, v)=\int_{\mathbb{R}}((-J \dot{u}, v)+(L(t) u, v)) d t, \quad \forall u, v \in E .
$$

It is easy to check that $Q(u, v)$ is a bounded quadratic form on $E$, and, hence, there exists a unique bounded self-adjoint operator $F: E \rightarrow E$ such that

$$
(F u, v)_{E}=Q(u, v), \quad \forall u, v \in E
$$

Besides, define a linear operator $K: L^{2} \rightarrow E$ by

$$
(K u, v)_{E}=(u, v)_{L^{2}}, \quad \forall u \in L^{2}, v \in E .
$$

In view of Lemma 1 , we know that $F$ is a Fredholm operator and $K$ is a compact operator.

Denote by $\mathscr{B}$ the set of all uniformly bounded symmetric $2 N \times 2 N$ matric functions. That is to say, $B \in \mathscr{B}$ if and only if $B^{T}(t)=B(t)$ for all $t \in \mathbb{R}$, and $B(t)$ is uniformly bounded in $t$ as the operator on $\mathbb{R}^{2 N}$. For any $B \in \mathscr{B}$, it is easy to see that $B$ determines a bounded self-adjoint operator on $L^{2}$, by $z(t) \mapsto B(t) z(t)$, for any $z \in L^{2}$, we still denote this operator by $B$ and then $K B: E \subset L^{2} \rightarrow E$ is a self-adjoint compact operator on $E$ and satisfies

$$
(K B u, v)_{E}=(B u, v)_{L^{2}}, \quad \forall u, v \in E .
$$

Before presenting the conditions on $R(t, z)$, we need the concept of Maslov index for homoclinic orbits introduced by Chen and $\mathrm{Hu}$ in [18] which is equivalent to the relative Morse index. We will give a brief introduction of it by Definition 7 , where for any $B \in \mathscr{B}$, we denote the associated index pair by $\left(\mu_{F}(K B), v_{F}(K B)\right)$.

Now we can present the conditions on $R(t, z)$ as follows. For notational simplicity, we set $B_{0}(t)=\nabla_{z}^{2} R(t, 0)$, and in what follows, the letter $c$ will be repeatedly used to denote various positive constants whose exact value is irrelevant. Besides, for two $2 N \times 2 N$ symmetric matrices $M_{1}$ and $M_{2}$, $M_{1} \leq M_{2}$ means that $M_{2}-M_{1}$ is semipositive definite.

$\left(R_{1}\right) R \in C^{2}\left(\mathbb{R} \times \mathbb{R}^{2 N}, \mathbb{R}\right)$, and there exists a constant $c>0$ such that

$$
\left|\nabla_{z}^{2} R(t, z)\right| \leq c, \quad \forall(t, z) \in \mathbb{R} \times \mathbb{R}^{2 N}
$$

$\left(R_{0}\right) \nabla_{z} R(t, 0) \equiv 0$ and $B_{0} \in \mathscr{B}$.

$\left(R_{\infty}\right)$ There exists some $R_{0}>0$ and continuous symmetric matrix functions $B_{1}, B_{2} \in \mathscr{B}$ with $\mu_{F}\left(K B_{1}\right)=$ $\mu_{F}\left(K B_{2}\right)$ and $v_{F}\left(K B_{2}\right)=0$ such that

$$
B_{1}(t) \leq \nabla_{z}^{2} R(t, z) \leq B_{2}(t), \quad \forall t \in \mathbb{R},|z|>R_{0} .
$$

Then, we have our first result.

Theorem 2. Assume that $\left(L_{1}\right),\left(R_{1}\right),\left(R_{0}\right)$, and $\left(R_{\infty}\right)$ hold. If

$$
\mu_{F}\left(K B_{1}\right) \notin\left[\mu_{F}\left(K B_{0}\right), \mu_{F}\left(K B_{0}\right)+v_{F}\left(K B_{0}\right)\right],
$$

then (HS) has at least one nontrivial homoclinic orbit. Moreover, if $v_{F}\left(K B_{0}\right)=0$ and $\left|\mu_{F}\left(K B_{1}\right)-\mu_{F}\left(K B_{0}\right)\right| \geq N$, the problem possesses at least two nontrivial homoclinic orbits.

Condition $\left(R_{\infty}\right)$ is a two-side pinching condition near the infinity, and we can relax $\left(R_{\infty}\right)$ to condition $\left(R_{\infty}^{ \pm}\right)$as follows.

$\left(R_{\infty}^{ \pm}\right)$There exist some $R_{0}>0$ and a continuous symmetric matrix function $B_{\infty} \in \mathscr{B}$ with $v_{F}\left(K B_{\infty}\right)=0$ such that

$$
\pm \nabla_{z}^{2} R(t, z) \geq \pm B_{\infty}(t), \quad \forall t \in \mathbb{R},|z|>R_{0} .
$$

Then, we have the following results.

Theorem 3. Assumes $\left(L_{1}\right),\left(R_{1}\right),\left(R_{0}\right),\left(R_{\infty}^{+}\right)$(or $\left.\left(R_{\infty}^{-}\right)\right)$, and $v_{F}\left(K B_{0}\right)=0$ hold. If $\mu_{F}\left(K B_{\infty}\right) \geq \mu_{F}\left(K B_{0}\right)+2\left(\operatorname{or} \mu_{F}\left(K B_{\infty}\right) \leq\right.$ $\left.\mu_{F}\left(K B_{0}\right)-2\right)$, then $(H S)$ has at least one nontrivial homoclinic orbit.

Theorem 4. Suppose that $\left(L_{1}\right),\left(R_{1}\right),\left(R_{0}\right),\left(R_{\infty}^{+}\right)\left(\right.$or $\left.\left(R_{\infty}^{-}\right)\right)$, and $v_{F}\left(K B_{0}\right)=0$ are satisfied. If in addition $R$ is even in $z$ and $\mu_{F}\left(K B_{\infty}\right) \geq \mu_{F}\left(K B_{0}\right)+2\left(\right.$ or $\left.\mu_{F}\left(K B_{\infty}\right) \leq \mu_{F}\left(K B_{0}\right)-2\right)$, then (HS) has at least $\left|\mu_{F}\left(K B_{\infty}\right)-\mu_{F}\left(K B_{0}\right)\right|-1$ pairs of nontrivial homoclinic orbits. 
Remark 5. Lemma 1 shows that $\sigma(A)$, the spectrum of $A$, consists of eigenvalues numbered by (counted in their multiplicities):

$$
\cdots \leq \lambda_{-2} \leq \lambda_{-1} \leq 0<\lambda_{1} \leq \lambda_{2} \leq \cdots
$$

with $\lambda_{ \pm k} \rightarrow \pm \infty$ as $k \rightarrow \infty$. Let $B_{0}(t) \equiv B_{0}$ and $B_{\infty}(t) \equiv$ $B_{\infty}$, with the constants $B_{0}, B_{\infty}$ satisfying $\lambda_{l}<B_{0}<\lambda_{l+1}$, and $\lambda_{l+i}<B_{\infty}<\lambda_{l+i+1}$ for some $l \in \mathbb{Z}$ and $i \geq 1$ (or $i \leq-1$ ). Define

$$
R(t, z)=\delta(|z|) \frac{1}{2} B_{0}|z|^{2}+(1-\delta(|z|)) \frac{1}{2} B_{\infty}|z|^{2},
$$

where $\delta$ is a smooth cutoff function satisfying $\delta(|z|)=$ $\left\{\begin{array}{l}1,|z|<1 \text {, } \\ 0,|z|>2\end{array}\right.$ By Proposition 12 that, it is easy to verify $R$ satisfies all the conditions in Theorem 2 . Furthermore, let the constant $B_{\infty}$ satisfy $\lambda_{l+i}<B_{\infty}<\lambda_{l+i+1}$ for some $l \in \mathbb{Z}$ and $i \geq 2$ (or $i \leq-2)$. Define

$$
R(t, z)=\delta(|z|) \frac{1}{2} B_{0}|z|^{2}+(1-\delta(|z|)) \frac{1}{2} B_{\infty}|z|^{2} .
$$

Then $R$ satisfies all the conditions in Theorems 3 and 4 . However, it is easy to see that some conditions of the main results in $[13-15,17]$ do not hold for these examples.

Remark 6. Note that the assumption $v_{F}\left(K B_{\infty}\right)=0$ in $\left(R_{\infty}^{ \pm}\right)$is not essential for our main results. For the case of $\left(R_{\infty}^{+}\right)$with $v_{F}\left(K B_{\infty}\right) \neq 0$, let $\widetilde{B}_{\infty}=B_{\infty}-\varepsilon I_{2 N}$ with $\varepsilon>0$ small enough, where $I_{2 N}$ is the identity map on $\mathbb{R}^{2 N}$; then, $\mu_{F}\left(K \widetilde{B}_{\infty}\right)=$ $\mu_{F}\left(K B_{\infty}\right)$ and $v_{F}\left(K \widetilde{B}_{\infty}\right)=0$, and, hence, $\left(R_{\infty}^{+}\right)$holds for $\widetilde{B}_{\infty}$. Therefore, Theorems 3 and 4 still hold in this case. While for the case of $\left(R_{\infty}^{-}\right)$with $v_{F}\left(K B_{\infty}\right) \neq 0$, if we replace $\mu_{F}\left(K B_{\infty}\right)$ by $\mu_{F}\left(K B_{\infty}\right)+v_{F}\left(K B_{\infty}\right)$ in Theorems 3 and 4 , then similar results hold. Indeed, let $\widetilde{B}_{\infty}=B_{\infty}+\varepsilon I_{2 N}$ with $\varepsilon>0$ small enough such that $\mu_{F}\left(K \widetilde{B}_{\infty}\right)=\mu_{F}\left(K B_{\infty}\right)+v_{F}\left(K B_{\infty}\right)$ and $v_{F}\left(K \widetilde{B}_{\infty}\right)=$ 0 , then this case is also reduced to the case of $\left(W_{\infty}^{-}\right)$for $\widetilde{B}_{\infty}$ with $v_{F}\left(K \widetilde{B}_{\infty}\right)=0$.

\section{Preliminaries}

In this section, we recall the definition of relative Morse index and saddle point reduction and give the relationship between them. For this propose, the notion of spectral flow will be used.

2.1. Relative Morse Index. Let $\mathscr{H}$ be a separable Hilbert space; for any self-adjoint operator $A$ on $\mathscr{H}$, there is a unique $A$ invariant orthogonal splitting

$$
\mathscr{H}=\mathscr{H}^{+}(A) \oplus \mathscr{H}^{-}(A) \oplus \mathscr{H}^{0}(A),
$$

where $\mathscr{H}^{0}(A)$ is the null space of $A, A$ is positive definite on $\mathscr{H}^{+}(A)$ and negative definite on $\mathscr{H}^{-}(A)$, and $P_{A}$ denotes the orthogonal projection from $\mathscr{H}$ to $\mathscr{H}^{-}(A)$. For any bounded self-adjoint Fredholm operator $\mathscr{F}$ and a compact self-adjoint operator $\mathscr{T}$ on $\mathscr{H}, P_{\mathscr{F}}-P_{\mathscr{F}-\mathscr{T}}$ is compact (see $\left[19\right.$, Lemma 2.7]), where $P_{\mathscr{F}}: \mathscr{H} \rightarrow \mathscr{H}^{-}(\mathscr{F})$ and $P_{\mathscr{F}-\mathscr{T}}:$ $\mathscr{H} \rightarrow \mathscr{H}^{-}(\mathscr{F}-\mathscr{T})$ are the respective projections. Then, by Fredholm operator theory, $\left.P_{\mathscr{F}}\right|_{\mathscr{H}^{-}(\mathscr{F}-\mathscr{T})}: \mathscr{H}^{-}(\mathscr{F}-\mathscr{T}) \rightarrow$ $\mathscr{H}^{-}(\mathscr{F})$ is a Fredholm operator. Here and in the sequel, we denote by ind $(\cdot)$ the Fredholm index of a Fredholm operator.

Definition 7. For any bounded self-adjoint Fredholm operator $\mathscr{F}$ and a compact self-adjoint operator $\mathscr{T}$ on $\mathscr{H}$, the relative Morse index pair $\left(\mu_{\mathscr{F}}(\mathscr{T}), v_{\mathscr{F}}(\mathscr{T})\right)$ is defined by

$$
\begin{aligned}
\mu_{\mathscr{F}}(\mathscr{T}) & =\operatorname{ind}\left(\left.P_{\mathscr{F}}\right|_{\mathscr{H}^{-}(\mathscr{F}-\mathscr{T})}\right), \\
v_{\mathscr{F}} & (\mathscr{T})=\operatorname{dim} \mathscr{H}^{0}(\mathscr{F}-\mathscr{T}) .
\end{aligned}
$$

2.2. Saddle Point Reduction. In this subsection, we describe the saddle point reduction in [20-22]. Recall that $\mathscr{H}$ is a real Hilbert space, and $A$ is a self-adjoint operator with domain $D(A) \subset \mathscr{H}$. Let $\Phi \in C^{1}(\mathscr{H}, \mathbb{R})$, with $\Phi^{\prime}(\theta)=0$. Assume that

(1) there exist real numbers $\alpha<\beta$ such that $\alpha, \beta \notin \sigma(A)$, and that $\sigma(A) \cap[\alpha, \beta]$ consists of at most finitely many eigenvalues of finite multiplicities;

(2) $\Phi^{\prime}$ is Gateaux differentiable in $\mathscr{H}$, which satisfies

$$
\left\|d \Phi^{\prime}(u)-\frac{\alpha+\beta}{2} I\right\| \leq \frac{\beta-\alpha}{2}, \quad \forall u \in \mathscr{H}
$$

and without loss of generality, we may assume that $\alpha=$ $-\beta, \beta>0$;

(3) $\Phi \in C^{2}(V, \mathbb{R}), V=D\left(|A|^{1 / 2}\right)$, with the norm

$$
\|z\|_{V}=\left(\left\||A|^{1 / 2} z\right\|_{\mathscr{H}}^{2}+\varepsilon^{2}\|z\|_{\mathscr{H}}^{2}\right)^{1 / 2}
$$

where $\varepsilon>0$ small and $-\varepsilon \notin \sigma(A)$.

Consider the solutions of the following equation:

$$
A z=\Phi^{\prime}(z), \quad z \in D(A) .
$$

Let

$$
\begin{gathered}
P_{0}=\int_{-\beta}^{\beta} d E_{\lambda}, \quad P_{+}=\int_{\beta}^{+\infty} d E_{\lambda}, \\
P_{-}=\int_{-\infty}^{-\beta} d E_{\lambda},
\end{gathered}
$$

where $\left\{E_{\lambda}\right\}$ is the spectral resolution of $A$, and let

$$
\mathscr{H}_{*}=P_{*} \mathscr{H}, \quad *=0, \pm .
$$

Decompose the space $V$ as follows:

$$
V=V_{0} \oplus V_{-} \oplus V_{+},
$$

where $V_{*}=\left|A_{\varepsilon}\right|^{-1 / 2} \mathscr{H}_{*}, *=0, \pm$, and $A_{\varepsilon}=A+\varepsilon I$.

For each $u \in \mathscr{H}$, we have the decomposition

$$
u=u_{+}+u_{0}+u_{-} \text {, }
$$


where $u_{*} \in \mathscr{H}_{*}$ and $*=0$, \pm ; let $z=z_{+}+z_{0}+z_{-}$, with

$$
z_{*}=\left|A_{\varepsilon}\right|^{-1 / 2} u_{*}, \quad *=0, \pm .
$$

Define a functional $f$ on $\mathscr{H}$ as follows:

$$
\begin{aligned}
f(u)= & \frac{1}{2}\left(\left\|u_{+}\right\|^{2}+Q_{+}\left\|u_{0}\right\|^{2}-Q_{-}\left\|u_{0}\right\|^{2}-\|u\|^{2}\right) \\
& -\Phi_{\varepsilon}(z),
\end{aligned}
$$

where $Q_{+}=\int_{0}^{\infty} d E_{\lambda}, Q_{-}=\int_{-\infty}^{0} d E_{\lambda}$, and $\Phi_{\varepsilon}(z)=(\varepsilon / 2)$ $\|z\|_{\mathscr{H}}+\Phi(z)$.

The Euler equation of this functional is the system

$$
\begin{gathered}
u_{ \pm}= \pm\left|A_{\varepsilon}\right|^{-1 / 2} P_{ \pm} \Phi_{\varepsilon}^{\prime}(z), \\
Q_{ \pm} u_{0}= \pm\left|A_{\varepsilon}\right|^{-1 / 2} Q_{ \pm} P_{0} \Phi_{\varepsilon}^{\prime}(z) .
\end{gathered}
$$

Thus, $z=z_{+}+z_{0}+z_{-}$is a solution of (21) if and only if $u=u_{+}+u_{0}+u_{-}$is a critical point of $f$. The implicit function can be applied, yielding a solution $z_{ \pm}\left(z_{0}\right)$ for fixed $z_{0} \in V_{0}$, such that $z_{ \pm} \in C^{1}\left(V_{0}, V_{ \pm}\right)$. Since $\operatorname{dim} V_{0}$ is finite, all topologies on $V_{0}$ are equivalent, and we choose $\|\cdot\|_{\mathscr{H}}$ as it norm. We have

$$
u_{ \pm}\left(z_{0}\right)=\left|A_{\varepsilon}\right|^{1 / 2} z_{ \pm}\left(z_{0}\right) \in C^{1}\left(\mathscr{H}_{0}, \mathscr{H}\right),
$$

which solves the system (28).

Let

$$
a\left(z_{0}\right)=f\left(u_{+}\left(z_{0}\right)+u_{-}\left(z_{0}\right)+u_{0}\left(z_{0}\right)\right),
$$

where $u_{0}\left(z_{0}\right)=\left|A_{\varepsilon}\right|^{1 / 2} z_{0}$, and let $z_{0}=x$ we have

$$
a(x)=\frac{1}{2}(A(z(x), z(x)))-\Phi(z(x)),
$$

where $z(x)=\xi(x)+x, \xi(x)=z_{+}(x)+z_{-}(x) \in D(A)$. Then, we have the following theorem due to Amann [20], Chang [21], and Long [22].

Theorem 8. Under assumptions (1), (2), and (3), there is a oneone correspondence

$$
x \longmapsto z=z(x)=z_{+}(x)+z_{-}(x)+x
$$

between the critical points of the $C^{2}$-function $a \in C^{2}\left(\mathscr{H}_{0}, \mathbb{R}\right)$ with the solutions of the operator equation

$$
A z=\Phi^{\prime}(z), \quad z \in D(A) .
$$

Moreover, the functional a satisfies

$$
\begin{aligned}
a(x) & =\frac{1}{2}(A(z(x), z(x)))-\Phi(z(x)), \\
a^{\prime}(x) & =A(z(x))-\Phi^{\prime}(z(x)) \\
& =A x-P_{0} \Phi^{\prime}(z(x)) \\
a^{\prime \prime}(x) & =\left[A-\Phi^{\prime \prime}(z(x))\right] z^{\prime}(x) \\
& =A P_{0}-P_{0} \Phi^{\prime \prime}(z(x)) z^{\prime}(x) .
\end{aligned}
$$

Since $\mathscr{H}_{0}$ is a finite dimensional space, for every critical point $x$ of $a$ in $\mathscr{H}_{0}$, the Morse index and nullity are finite, and we denote them by $\left(m_{a}^{-}(x), m_{a}^{0}(x)\right)$.

Now, let the Hilbert space $\mathscr{H}$ be $L^{2}\left(\mathbb{R}, \mathbb{R}^{2 N}\right)$, and the operator $A$ be $\widetilde{F}=-J(d / d t)+L, \Phi(u)=\int_{-\infty}^{\infty} R(t, u)$. Then we have $V=E$. For $R \in C^{2}\left(\mathbb{R} \times \mathbb{R}^{2 N}, \mathbb{R}\right)$ and $\left|\nabla_{z}^{2} R\right| \leq C_{R}$, for all $(t, z) \in$ $\mathbb{R} \times \mathbb{R}^{2 N}$, let $-\alpha=\beta \geq 2\left(C_{R}+1\right)$ and $\beta \notin \sigma(\widetilde{F})$; we have $A$ and $\Phi$ satisfying the previous conditions. Thus, from Theorem 8 , we can solve our problems on the finite dimensional space. Similar to Lemma 2.2 and Remark 2.3 in [23], we have the following estimates.

Lemma 9. Assume that $R \in C^{2}\left(\mathbb{R} \times \mathbb{R}^{2 N}, \mathbb{R}\right),\left|\nabla_{z}^{2} R\right| \leq C_{R}$, for all $(t, z) \in \mathbb{R} \times \mathbb{R}^{2 N}$ and $\nabla_{z} R(t, 0) \equiv 0$; then one has

$$
\left\|u^{ \pm}(x)\right\|_{L^{2}} \leq \frac{2 \sqrt{\beta}\left(C_{R}+1\right)}{\beta-2 C_{R}-3 \varepsilon}\|x\|_{L^{2}}, \quad \forall x \in \mathscr{H}_{0} .
$$

Moreover, one has

$$
\left\|\left(u^{ \pm}\right)^{\prime}(x)\right\|_{L^{2}} \longrightarrow 0, \quad \beta \longrightarrow \infty .
$$

Proof. Note that

$$
u^{ \pm}(x)= \pm\left|A_{\varepsilon}\right|^{-1 / 2} P_{ \pm} \Phi_{\varepsilon}^{\prime}\left(z^{+}+z^{-}+x\right) .
$$

From $\nabla_{z} R(t, 0)=0,\left|\nabla_{z}^{2} R\right| \leq C_{R}$, we have $\Phi^{\prime}(0)=0$ and $\left\|\Phi^{\prime}(z)\right\|_{L^{2}} \leq C_{R}\|z\|_{L^{2}}$. Since $\left\|\left|A_{\varepsilon}\right|^{-1 / 2} P_{ \pm}\right\| \leq 1 / \sqrt{(\beta-\varepsilon)}$, we have

$$
\begin{aligned}
& \left\|u^{ \pm}(x)\right\|_{L^{2}} \\
& \leq \frac{1}{\sqrt{\beta-\varepsilon}}\left\|\Phi^{\prime}\left(z^{+}+z^{-}+x\right)+\varepsilon\left(z^{+}+z^{-}+x\right)\right\|_{L^{2}} \\
& \quad \leq \frac{C_{R}+\varepsilon}{\sqrt{\beta-\varepsilon}}\left\|z^{+}+z^{-}+x\right\|_{L^{2}} \\
& \quad \leq \frac{C_{R}+\varepsilon}{\sqrt{\beta-\varepsilon}}\left(\frac{\left\|u^{+}(x)\right\|_{L^{2}}}{\sqrt{\beta}}+\frac{\left\|u^{-}(x)\right\|_{L^{2}}}{\sqrt{\beta}}+\|x\|_{L^{2}}\right) .
\end{aligned}
$$

Therefore,

$$
\left\|u^{+}(x)\right\|_{L^{2}}+\left\|u^{-}(x)\right\|_{L^{2}} \leq \frac{2 \sqrt{\beta}\left(C_{R}+\varepsilon\right)}{\beta-2 C_{R}-3 \varepsilon}\|x\|_{L^{2}} .
$$

Next, since

$$
\begin{aligned}
\left(u^{ \pm}\right)^{\prime}(x)= & \pm\left|A_{\varepsilon}\right|^{-1 / 2} P_{ \pm} \Phi_{\varepsilon}^{\prime} \\
& \times\left(z^{+}+z^{-}+x\right)\left(\left(z^{+}\right)^{\prime}(x)+\left(z^{-}\right)^{\prime}(x)+I\right),
\end{aligned}
$$

where $I$ is the identity map on $\mathscr{H}_{0}$, we have

$$
\begin{aligned}
& \left\|\left(u^{+}\right)^{\prime}(x)\right\|_{L^{2}}+\left\|\left(u^{-}\right)^{\prime}(x)\right\|_{L^{2}} \\
& \quad \leq \frac{2 \sqrt{\beta}\left(C_{R}+\varepsilon\right)}{\beta-2 C_{R}-3 \varepsilon}, \quad \forall x \in \mathscr{H}_{0} .
\end{aligned}
$$


Remark 10. For $z(x)$, we also have that there is a constant $C>$ 0 dependent of $C_{R}$, but independent of $\beta$, such that

$$
\left\|z^{ \pm}(x)\right\|_{V} \leq \frac{C}{\sqrt{\beta}}\|x\|_{L^{2}}, \quad\left\|z^{\prime \pm}(x)\right\|_{V} \leq \frac{C}{\sqrt{\beta}}, \quad \forall x \in \mathscr{H}_{0} .
$$

If $R$ satisfies the condition $\left(R_{1}\right)$, then for any homoclinic orbit $z$ of (HS), $\nabla_{z}^{2} R(\cdot, z) \in \mathscr{B}$, and, hence, we have the associated index pair $\left(\mu_{F}(K B), v_{F}(K B)\right)$. For notation simplicity, in what follows, we set

$$
\begin{aligned}
& \mu_{F}(z)=\mu_{F}\left(K \nabla_{z}^{2}(R(t, z))\right), \\
& v_{F}(z)=v_{F}\left(K \nabla_{z}^{2}(R(t, z))\right) .
\end{aligned}
$$

Theorem 11. Let $R \in C^{2}\left(\mathbb{R} \times \mathbb{R}^{2 N}, \mathbb{R}\right)$ satisfying $\left|\nabla_{z}^{2} R\right| \leq C_{R}$, for all $(t, z) \in \mathbb{R} \times \mathbb{R}^{2 N}$ and $\nabla_{z} R(t, 0) \equiv 0$. For each critical point $x$ of a in $\mathscr{H}_{0}, z(x)$ is a homoclinic orbit of $(H S)$ and one has

$$
\begin{gathered}
m_{a}^{-}(x)=\operatorname{dim}\left(E^{-}\left(\mathscr{H}_{0}\right)\right)+\mu_{F}\left(K \nabla_{z}^{2} R(t, z(x))\right) \\
=\operatorname{dim}\left(E^{-}\left(\mathscr{H}_{0}\right)\right)+\mu_{F}(z(x)), \\
m_{a}^{0}(x)=v_{F}\left(K \nabla_{z}^{2} R(t, z(x))=v_{F}(z(x)),\right.
\end{gathered}
$$

where $\operatorname{dim}\left(E^{-}\left(\mathscr{H}_{0}\right)\right)$ is the dimension of the space $\int_{-\beta}^{0^{-}} d E_{\lambda}$ $\left(\mathscr{H}_{0}\right)$.

This theorem shows the relations between the relative Morse index and the Morse index of the saddle point reduction, and it will play an important role in the proof of our main results. The proof of this theorem will be postponed to the next subsection where the notion of spectral flow will be used.

2.3. The Relationship between $\mu_{F}(T)$, Spectral Flow, and the Morse Index of Saddle Point Reduction. It is well known that the concept of spectral flow was first introduced by Atiyah et al. in [24] and then extensively studied in [19, 25-28]. Here, we give a brief introduction of the spectral flow as introduced in [18]. Let $\mathscr{H}$ be a separable Hilbert space as defined before, and $\left\{\mathscr{F}_{\theta} \mid \theta \in[0,1]\right\}$ be a continuous path of self-adjoint Fredholm operators on the Hilbert space $\mathscr{H}$. The spectral flow of $\mathscr{F}_{\theta}$ represents the net change in the number of negative eigenvalues of $\mathscr{F}_{\theta}$ as $\theta$ runs from 0 to 1 , where the counting follows from the rule that each negative eigenvalue crossing to the positive axis contributes +1 and each positive eigenvalue crossing to the negative axis contributes -1 , and for each crossing, the multiplicity of eigenvalue is taken into account. In the calculation of spectral flow, a crossing operator introduced in [28] will be used. Take a $C^{1}$ path $\left\{\mathscr{F}_{\theta} \theta \mid \in[0,1]\right\}$ and let $\mathscr{P}_{\theta}$ be the projection from $\mathscr{H}$ to $\mathscr{H}^{0}\left(\mathscr{F}_{\theta}\right)$. When eigenvalue crossing occurs at $\mathscr{F}_{\theta}$, the operator

$$
\mathscr{P}_{\theta} \frac{\partial}{\partial \theta} \mathscr{F}_{\theta} \mathscr{P}_{\theta}: \mathscr{H}^{0}\left(\mathscr{F}_{\theta}\right) \longrightarrow \mathscr{H}^{0}\left(\mathscr{F}_{\theta}\right)
$$

is called a crossing operator, denoted by $C_{r}\left[\mathscr{F}_{\theta}\right]$. As mentioned in [28], an eigenvalue crossing at $\mathscr{F}_{\theta}$ is said to be regular if the null space of $C_{r}\left[\mathscr{F}_{\theta}\right]$ is trivial. In this case, we define

$$
\operatorname{sign} C_{r}\left[\mathscr{F}_{\theta}\right]=\operatorname{dim} \mathscr{H}^{+}\left(C_{r}\left[\mathscr{F}_{\theta}\right]\right)-\operatorname{dim} \mathscr{H}^{-}\left(C_{r}\left[\mathscr{F}_{\theta}\right]\right) .
$$

A crossing occurring at $\mathscr{F}_{\theta}$ is called simple crossing if $\operatorname{dim} \mathscr{H}^{0}\left(\mathscr{F}_{\theta}\right)=1$.

As indicated in [19], the spectral flow $\operatorname{Sf}\left(\mathscr{F}_{\theta}\right)$ will remain the same after a small disturbance of $\mathscr{F}_{\theta}$, that is, $\operatorname{Sf}\left(\mathscr{F}_{\theta}\right)=$ $\operatorname{Sf}\left(\mathscr{F}_{\theta}+\varepsilon\right.$ id $)$ for $\varepsilon>0$ and small enough, where id is the identity map on $\mathscr{H}$. Furthermore, we can choose suitable $\varepsilon$ such that all the eigenvalue crossings occurring in $\mathscr{F}_{\theta}, 0 \leq$ $\theta \leq 1$ are regular [28]. Thus, without loss of generality, we may assume that all the crossings are regular. Let $\mathscr{D}$ be the set containing all the points in $[0,1]$ at which the crossing occurs. The set $\mathscr{D}$ contains only finitely many points. The spectral flow of $\mathscr{F}_{\theta}$ is

$$
\begin{aligned}
\operatorname{Sf} & \left(\mathscr{F}_{\theta}, 0 \leq \theta \leq 1\right) \\
= & \sum_{\theta \in \mathscr{D}^{*}} \operatorname{sign} C_{r}\left[\mathscr{F}_{\theta}\right]-\operatorname{dim} \mathscr{H}^{-}\left(C_{r}\left[\mathscr{F}_{0}\right]\right) \\
& +\operatorname{dim} \mathscr{H}^{+}\left(C_{r}\left[\mathscr{F}_{1}\right]\right),
\end{aligned}
$$

where $\mathscr{D}^{*}=\mathscr{D} \cap(0,1)$. In what follows, the spectral flow of $\mathscr{F}_{\theta}$ will be simply denoted by $\operatorname{Sf}\left(\mathscr{F}_{\theta}\right)$ when the starting and end points of the flow are clear from the contents. And $P_{\mathscr{F}_{\theta}}$ will be simply denoted by $P_{\theta}$.

Proposition 12 (see [18, Proposition 3]). Suppose that, for each $\theta \in[0,1], \mathscr{F}_{\theta}-\mathscr{F}_{0}$ is a compact operator on $\mathscr{H}$ then

$$
\text { ind }\left(\left.P_{0}\right|_{\mathscr{H}^{-}\left(\mathscr{F}_{1}\right)}\right)=-S f\left(\mathscr{F}_{\theta}\right) \text {. }
$$

Thus, from Definition 7,

$$
\mu_{\mathscr{F}_{0}}(\mathscr{T})=-\operatorname{Sf}\left(\mathscr{F}_{\theta}, 0 \leq \theta \leq 1\right),
$$

where $\mathscr{F}_{\theta}=\mathscr{F}-\theta \mathscr{T}, \mathscr{T}$ is a compact operator. Moreover, if $\sigma(\mathscr{T}) \subset[0, \infty)$ and $0 \notin \sigma_{P}(\mathscr{T})$, from the definition of spectral flow, we have

$$
\begin{aligned}
\mu_{\mathscr{F}_{0}}(\mathscr{T}) & =-\operatorname{Sf}\left(\mathscr{F}_{\theta}, 0 \leq \theta \leq 1\right) \\
& =\sum_{\theta \in[0,1)} v_{\mathscr{F}}(\theta \mathscr{T}) \\
& =\sum_{\theta \in[0,1)} \operatorname{dim} \mathscr{H}^{0}(\mathscr{F}-\theta \mathscr{T}) .
\end{aligned}
$$

The proof of Theorem 11 is the direct consequence of the aformentioned Proposition 12 and Theorem 3.2 in [19], so we omit it here.

Remark 13. The case of $R_{\infty}^{-}$can be transformed into the case of $R_{\infty}^{+}$. More concretely, the $R_{\infty}^{-}$case follows from the $R_{\infty}^{+}$ case by applying to the function $\widetilde{R}(t, z)=-R(-t, z)$. If $z(t)$ 
is a homoclinic solution of $\widetilde{F} z(t)=\nabla_{z} R(t, z(t))$, let $\widetilde{z}(t)=$ $z(-t)$, it is easy to check that $\widetilde{z}(t)$ is a homoclinic solution of $\widetilde{F} \widetilde{z}(t)=\nabla_{z} \widetilde{R}(t, \widetilde{z}(t))$, and this is a one-one correspondence between the two systems. By the definition of spectral flow and it is catenation property [19], we have $\mu_{F}\left(-B_{\infty}(-t)\right)-$ $\mu_{F}\left(-B_{0}(-t)\right)=\mu_{F}\left(B_{0}(t)\right)-\mu_{F}\left(B_{\infty}(t)\right)$. Thus, we only consider the case of $R_{\infty}^{+}$from now on.

\section{Proof of Our Main Results}

Proof of Lemma 1. Recall the operator $\widetilde{F}=-\mathscr{J}(d / d t)+L(t)$, with domain $D(\widetilde{F})=H^{1}\left(\mathbb{R}, \mathbb{R}^{2 N}\right)$ if $L(t)$ is bounded and $D(\widetilde{F}) \subset H^{1}\left(\mathbb{R}, \mathbb{R}^{2 N}\right)$ if $L(t)$ is unbounded. $D(\widetilde{F})$ is a Hilbert space equipped with the norm $\|z\|_{\widetilde{F}}=\|(I+|\widetilde{F}|) z\|_{L^{2}}$, for all $z \in D(\widetilde{F})$. Recall the Hilbert space $E=D\left(|\widetilde{F}|^{1 / 2}\right)$, with the inner product and norm by

$$
\begin{gathered}
(u, v)_{E}=\left(|\widetilde{F}|^{1 / 2} u,|\widetilde{F}|^{1 / 2} v\right)_{2}+(u, v)_{2}, \\
\|u\|_{E}=(u, u)_{E}^{1 / 2}
\end{gathered}
$$

where $(\cdot, \cdot)_{L^{2}}$ denotes the usual inner product on $L^{2}\left(\mathbb{R}, \mathbb{R}^{2 N}\right)$. From $\left(L_{1}\right)$, there is a matrix $L_{0}$ such that $P\left(L(t)-L_{0}\right) \geq 0$ for all $t \in \mathbb{R}$. We have $L(t)=\widetilde{F}-\widetilde{F}_{0}+L_{0}$, with $\widetilde{F}_{0}=-\mathscr{J}(d / d t)+L_{0}$ and $D\left(\widetilde{F}_{0}\right)=H^{1}$. Thus, for any $z \in E$,

$$
\begin{aligned}
& \left|\left(L(t) z, P^{T} z\right)_{L^{2}}\right| \\
& \quad \leq\left|\left(\widetilde{F} z, P^{T} z\right)_{L_{2}}\right|+\left|\left(\widetilde{F}_{0} z, P^{T} z\right)_{L_{2}}\right|+\left|\left(L_{0} z, P^{T} z\right)_{L_{2}}\right| \\
& \quad \leq c\|z\|_{E}^{2} .
\end{aligned}
$$

Let $K \subset E$ be a bounded set. We will show that $K$ is precompact in $L^{p}$ for $1 \leq p \in(2 /(1+\alpha), \infty)$. We divide the proof into three steps.

Step 1 (the case of $p=2$ ). For $R>0$, from $\left(L_{1}\right)$ and (53) we have

$$
\begin{aligned}
\int_{|t|>R}|z|^{2} d t & \leq c|R|^{\alpha-2} \int_{|t|>R}\left\langle L(t) z, P^{T} z\right\rangle_{\mathbb{R}^{2 N}} d t \\
& \leq c|R|^{\alpha-2}\|z\|_{E}^{2} .
\end{aligned}
$$

For any $\varepsilon>0$, from (54), we can choose $R_{0}$ large enough, such that

$$
\int_{|t|>R_{0}}|z|^{2} d t<\frac{\varepsilon^{2}}{4}, \quad \forall z \in K .
$$

On the other hand, by the definition of $\|\cdot\|_{E}$, we have

$$
\int_{|t| \leq R_{0}}|z|^{2} d t \leq\|z\|_{E} \leq C, \quad \forall z \in K .
$$

Thus, by the Sobolev compact embedding theorem, there exist $z_{1}, z_{2}, \ldots, z_{m} \in K$, such that for any $z \in K$, there is $z_{i}$ satisfying

$$
\left\|z-z_{i}\right\|_{L^{p}\left(\left(-R_{0}, R_{0}\right), \mathbb{R}^{2 N}\right)}^{p}<\frac{\varepsilon^{2}}{2} .
$$

From (55) and (57), we have $\left\|z-z_{i}\right\|_{L^{2}}<\varepsilon$; thus, $K$ has a finite $\varepsilon$-net in $L^{2}$, and so the embedding $E \hookrightarrow L^{2}$ is compact.

Step 2 (the case of $p>2$ ). Since $E$ is continuously embedded in $H^{1 / 2}$, hence, by the Sobolev embedding theorem, $E$ is continuously embedded in $L^{p}$, for all $p>2$. For any $p>2$, by the Hölder inequality we have

$$
\int_{\mathbb{R}}|z|^{p} d t \leq\|z\|_{L^{2}}\|z\|_{L^{2(p-1)}}^{p-1} \leq C\|z\|_{L^{2}}\|z\|_{E}^{p-1},
$$

thus, the embedding $E \hookrightarrow L^{p}$ is compact, for all $p>2$.

Step 3 (the case of $1 \leq p \in(2 /(1+\alpha), 2))$. First, we have $(\alpha /(2-p)) \cdot p>1$; so we can choose $\alpha_{p}$ satisfying $\alpha_{p} \in(0, \alpha)$ and $\left(\alpha_{p} /(2-p) \cdot p\right)>1$. Denote that $r=\alpha_{p} /(2-p)$. For $R>0$ and $z \in E$, denote that $E_{R}^{1}(z)=\left\{t ;|t| \geq R\right.$ and $\left.|t|^{r}|z(t)|>1\right\}$ and $E_{R}^{2}(z)=\left\{t ;|t| \geq R\right.$ and $\left.|t|^{r}|z(t)| \leq 1\right\}$. Then, from (53),

$$
\begin{aligned}
\int_{E_{R}^{1}(z)}|z|^{p} d t & =\int_{E_{R}^{1}(z)}\left(|t|^{r}|z|\right)^{p}|t|^{-r p} d t \\
& \leq \int_{E_{R}^{1}(z)}|z|^{2}|t|^{\alpha_{p}} d t \\
& \leq \frac{c}{|R|^{\alpha-\alpha_{p}}}\left|\left(L(t) z, P^{T} z\right)_{L^{2}}\right| \\
& \leq \frac{c}{|R|^{\alpha-\alpha_{p}}}\|z\|_{E}^{2},
\end{aligned}
$$

and so

$$
\begin{aligned}
& \int_{|t| \geq R}|z|^{p} d t \\
& \quad=\int_{E_{R}^{1}(z)}|z|^{p} d t+\int_{E_{R}^{2}(z)}|z|^{p} d t \\
& \leq \frac{c}{|R|^{\alpha-\alpha_{p}}}\|z\|_{E}^{2}+\frac{2}{(r p-1) R^{r p-1}}, \quad \forall z \in E .
\end{aligned}
$$

Let $K \subset E$ be a bounded set. For any $\varepsilon>0$, from (60), choose $R_{0}>0$ large enough, such that

$$
\int_{|t| \geq R_{0}}|z|^{p} d t<\frac{\varepsilon^{p}}{4}, \quad \forall z \in K .
$$

On the other hand, by the Sobolev compact embedding theorem, there are $z_{1}, z_{2}, \ldots, z_{m} \in K$, such that for any $z \in K$, there exists $z_{i}$ satisfying

$$
\left\|z-z_{i}\right\|_{L^{p}\left(\left(-R_{0}, R_{0}\right), \mathbb{R}^{2 N}\right)}^{p}<\frac{\varepsilon^{p}}{2} .
$$

From (61) and (62), we have

$$
\left\|z-z_{i}\right\|_{L^{p}}<\varepsilon
$$

that is to say, $K$ has a finite $\varepsilon$-net in $L^{p}$, and the embedding $E \hookrightarrow L^{p}$ is compact. The proof of the lemma is complete. 
Consider the homoclinic orbits of the linear Hamiltonian systems

$$
\begin{gathered}
\dot{z}(t)=J B(t) z(t), \quad \forall t \in \mathbb{R}, \\
z(t) \longrightarrow 0, \quad|t| \longrightarrow \infty
\end{gathered}
$$

where $z(t): \mathbb{R} \rightarrow \mathbb{R}^{2 N}, J=\left(\begin{array}{cc}0 & -I_{N} \\ I_{N} & 0\end{array}\right)$, and $B(t)$ is a continuous symmetric matrix function. Denote by $S$ the set of homoclinic orbits of linear systems (64); then, $S$ is a linear subspace of $L^{2}\left(\mathbb{R}, \mathbb{R}^{2 N}\right)$ and we have the following lemma.

Lemma 14. The dimension of the solution space $S$ will be less than or equal to $N$. Thus for any homoclinic orbit $z(t)$ of $(H S)$, if $R$ satisfies $\left(R_{1}\right)$, one has

$$
0 \leq v_{F}(z) \leq N
$$

Proof. As usual, we define the symplectic groups on $\mathbb{R}^{2 N}$ by

$$
S p(2 N)=\left\{M \in \mathscr{L}\left(\mathbb{R}^{2 N}\right), \mid M^{T} J M=J\right\},
$$

where $\mathscr{L}\left(\mathbb{R}^{2 N}\right)$ is the set of all $2 N \times 2 N$ real matrices, and $M^{T}$ denotes the transpose of $M$. Let $W(t)$ be the fundamental solution of (64); then, $W(t)$ is a path in $S p(2 N)$. Let $z(t)$ be a nontrivial homoclinic orbit of (64); that is to say, $z(0) \neq 0$ and satisfies

$$
\begin{aligned}
& z(t)=W(t) z(0), \\
& \lim _{t \rightarrow \infty} W(t) z(0)=0 .
\end{aligned}
$$

Denote by $S_{0}$ the subset of $\mathbb{R}^{2 N}$ satisfying

$$
S_{0}=\left\{z \in \mathbb{R}^{2 N} \mid \lim _{t \rightarrow \infty} W(t) z=0\right\},
$$

Then, we have $\operatorname{dim} S=\operatorname{dim}\left(S_{0}\right)$. We claim that $J z_{0} \notin S_{0}$ if $z_{0} \in$ $S_{0}$ and $z_{0} \neq 0$. We prove it indirectly. Assume that $z_{0}, J z_{0} \in S_{0}$ with $z_{0} \neq 0$; that is to say,

$$
\begin{aligned}
& \lim _{t \rightarrow \infty} W(t) z_{0}=0, \\
& \lim _{t \rightarrow \infty} W(t) J z_{0}=0 .
\end{aligned}
$$

Since $W(t)$ is a path in $\operatorname{Sp}(2 N), W^{T}(t) J W(t)=J$, for all $t \in \mathbb{R}$, thus

$$
\begin{aligned}
0 & =\lim _{t \rightarrow \infty}\left(J W(t) z_{0}, W(t) J z_{0}\right)_{\mathbb{R}^{2 N}} \\
& =-\lim _{t \rightarrow \infty}\left(z_{0}, W^{T}(t) J W(t) J z_{0}\right)_{\mathbb{R}^{2 N}} \\
& =\left(z_{0}, z_{0}\right)_{\mathbb{R}^{2 N}},
\end{aligned}
$$

which contradicts $z_{0} \neq 0$. Since $J$ is an isomorphism on $\mathbb{R}^{2 N}$, we have $\operatorname{dim} S_{0} \leq N$. And from the definition of $v_{F}(z)$ in the last part of Section 2.2, we have completed the proof.

Before the proof of Theorem 2, we need the following lemma. Since $R$ satisfies condition $\left(R_{1}\right)$, performing on (HS) the saddle point reduction, choose a suitable number $\beta$, which is used in the projection for the saddle point reduction in Section 2.2. Let

$$
\begin{gathered}
P=\int_{-\beta}^{\beta} d E_{\lambda}, \\
X=P L^{2}\left(\mathbb{R}, \mathbb{R}^{2 N}\right) .
\end{gathered}
$$

By Theorem 8 , we have a functional $a(x)$ with $x \in X$, whose critical points give rise to solutions of (HS).

Lemma 15. (1) a satisfies (PS) condition;

(2) $H_{q}(X, a ; \mathbb{R}) \cong \delta_{q, r} \mathbb{R}, q=0,1, \ldots$ for $-a \in \mathbb{R}$ large enough, where $r=\operatorname{dim}\left(E^{-}(X)\right)+\mu_{F}\left(K B_{1}\right)$.

Proof. Assume that there is a sequence $\left\{x_{n}\right\} \subset X$, satisfying $a^{\prime}\left(x_{n}\right) \rightarrow 0(n \rightarrow \infty)$. That is,

$$
\left\|F z_{n}-K \nabla_{z} R\left(t, z_{n}\right)\right\|_{E} \longrightarrow 0,
$$

where $z_{n}=z\left(x_{n}\right)$ defined in Section 2.1. Since $X$ is a finite dimensional space, and from the definition of $z_{n}$, it is enough to prove that $\left\{z_{n}\right\}$ is bounded in $E$. For each $\varepsilon \in(0,1)$, define $C_{n} \in \mathscr{B}$ by

$$
C_{n}(t)= \begin{cases}\int_{0}^{1} \nabla_{z}^{2} R\left(t, s z_{n}\right) d s, & \left|z_{n}(t)\right| \geq \frac{R_{0}}{\varepsilon}, \\ B_{1}(t), & \left|z_{n}(t)\right|<\frac{R_{0}}{\varepsilon} .\end{cases}
$$

It is easy to verify that $\left\{C_{n}\right\}$ satisfies

$$
\begin{aligned}
& B_{1}(t)-\varepsilon\left(B_{1}(t)+c \cdot I\right) \\
& \quad \leq C_{n}(t) \leq B_{2}(t)+\varepsilon\left(c \cdot I-B_{2}(t)\right), \quad \forall t \in \mathbb{R},
\end{aligned}
$$

where $c$ is the constant in condition $\left(R_{1}\right)$ and $I$ is the identity map on $\mathbb{R}^{2 N}$. Since $B_{1} \leq B_{2}, \mu_{F}\left(B_{1}\right)=\mu_{F}\left(B_{2}\right)$ and $v_{F}\left(K B_{1}\right)=$ $v_{F}\left(K B_{2}\right)=0$, we can choose $\varepsilon$ small enough, such that for each $n \in \mathbb{N}^{+}, \mu_{F}\left(K C_{n}\right)=\mu_{F}\left(K B_{1}\right)$ and $v_{F}\left(K C_{n}\right)=0$. Thus $F-K C_{n}$ is reversible on $E$, and there is a constant $\delta>0$, such that

$$
\left\|\left(F-K C_{n}\right) z\right\|_{E} \geq \delta\|z\|_{E}, \quad \forall z \in E, n \in \mathbb{N}^{+} .
$$

On the other hand, for $b \in(0,1)$, there is a constant $c>0$ depending on $b$, such that for each $n \in \mathbb{N}^{+}$,

$$
\left|\nabla_{z} R\left(t, z_{n}(t)\right)-C_{n} z_{n}(t)\right| \leq c\left|z_{n}(t)\right|^{b}, \quad \forall t \in \mathbb{R} .
$$

Choose $b>(1-\alpha) /(1+\alpha)$ in (76); that is, $1+b \in(2 /(1+\alpha), 2)$, we have

$$
\begin{aligned}
& \left\|\left(F z_{n}-K \nabla_{z} R\left(t, z_{n}\right)\right)-\left(F-K C_{n}\right) z_{n}\right\|_{E}^{2} \\
& \quad=\left\|K\left(\nabla_{z} R\left(t, z_{n}\right)-C_{n} z_{n}\right)\right\|_{E}^{2} \\
& \quad \leq\left\|\nabla_{z} R\left(t, z_{n}\right)-C_{n} z_{n}\right\|_{L^{2}}^{2} \\
& \quad \leq c \int_{\mathbb{R}} \frac{\left|\nabla_{z} R\left(t, z_{n}\right)-C_{n} z_{n}\right|}{\left|z_{n}\right|^{b}}\left|z_{n}\right|^{1+b} d t \\
& \quad \leq c\left\|z_{n}\right\|_{L^{1+b}}^{1+b} .
\end{aligned}
$$


As we claimed in the introduction, in (76) and (77), the letter $c$ denotes different positive constants whose exact value is irrelevant. Thus, from (72), (75), (77), and Lemma 1, we have that $\left\{z_{n}\right\}$ is bounded in $E$, and $a$ satisfies the (PS) conditions. And by Lemma 5.1 in Chapter II of [21], we have

$$
H_{q}\left(X,(a)_{\alpha} ; \mathbb{R}\right) \cong \delta_{q, r} \mathbb{R}, \quad q=0,1, \ldots,
$$

for $-\alpha \in \mathbb{R}$ large enough.

From Theorem 11, Lemmas 14 and 15, Theorem 2 is a direct consequence of Theorem 5.1 and Corollary 5.2 in Chapter II of [21].

In order to proof Theorems 3 and 4, we need the following lemma which is similar to Lemma 3.4 in [29] and Lemma 3.3 in [23].

Lemma 16. Assume that $\left(R_{1}\right),\left(R_{0}\right)$, and $\left(R_{\infty}^{+}\right)$hold; then, there exists a sequence of functions $R_{k} \in C^{2}\left(\mathbb{R} \times \mathbb{R}^{2 N}, \mathbb{R}\right), k \in \mathbb{N}$, satisfying the following properties.

(1) There exists an increasing sequence of real numbers $M_{k} \rightarrow \infty(k \rightarrow \infty)$ such that

$$
R_{k}(t, z) \equiv R(t, z), \quad \forall t \in \mathbb{R},|z| \leq M_{k} .
$$

(2) For each $k \in \mathbb{N}$, there is a $C>0$ independent of $k$, such that

$$
\begin{aligned}
& \left|\nabla_{z}^{2} R_{k}(t, z)\right| \leq C, \quad \forall t \in \mathbb{R}, z \in \mathbb{R}^{2 N}, \\
& \nabla_{z}^{2} R_{k}(t, z) \geq B_{\infty}, \quad \forall t \in \mathbb{R},|z| \geq R_{0} .
\end{aligned}
$$

(3) For each $k \in \mathbb{N}$, there exist some $C_{k}>0$ and a constant $\gamma$ with $\gamma I_{2 N}>B_{\infty}, v_{F}\left(K \gamma I_{2 N}\right)=0$, such that

$$
\left|\nabla_{z} R_{k}(t, z)-\gamma z\right|<C_{k}, \quad \forall(t, z) \in \mathbb{R} \times \mathbb{R}^{2 N},
$$

where $I_{2 N}$ is the identity map on $\mathbb{R}^{2 N}$.

Proof. Define $\eta:[0, \infty) \rightarrow \mathbb{R}$ by

$$
\eta(s)= \begin{cases}0, & 0 \leq s<1, \\ \frac{2}{9}(s-1)^{3}-\frac{1}{9}(s-1)^{4}, & 1 \leq s<2, \\ 1-\frac{128}{9\left(12+s^{2}\right)}, & 2 \leq s<\infty .\end{cases}
$$

It is easy to see that $\eta \in C^{2}([0, \infty), \mathbb{R})$. Choose a sequence $\left\{M_{k}\right\}$ of positive numbers such that $R_{0}<M_{1}<M_{2}<\cdots<$ $M_{k}<\cdots \rightarrow \infty$ as $k \rightarrow \infty$. For each $k \in \mathbb{N}$, let $\eta_{k}(s)=$ $\eta\left(s / M_{k}\right)$ and

$$
R_{k}(t, z)=\left(1-\eta_{k}(|z|)\right) R(t, z)+\frac{\gamma}{2} \eta_{k}(|z|)|z|^{2}, \quad k \in \mathbb{N} .
$$

As in $[23,29]$, we can check that $R_{k}$ satisfies (79)-(81) for each $k \in \mathbb{N}$.
For each $k \in \mathbb{N}$, we consider the following problem

$$
\begin{gathered}
\tilde{F} z=\nabla_{z} R_{k}(t, z), \\
z(t) \longrightarrow 0, \quad z^{\prime}(t) \longrightarrow 0, \quad t \longrightarrow \infty,
\end{gathered}
$$

where $R_{k}$ is given in Lemma 16. Performing on (HS) $k$ the saddle point reduction, we choose the number $\beta$ which is used in the projection for the saddle point reduction in Section 2.2. First, we choose

$$
\beta>\max \{2(C+1), 2(\gamma+1)\}, \quad \beta \notin \sigma\left(A_{0}\right) .
$$

Let

$$
\begin{gathered}
P_{\beta}=\int_{-\beta}^{\beta} d E_{\lambda}, \\
X_{\beta}=P_{\beta} L^{2}\left(\mathbb{R}, \mathbb{R}^{2 N}\right) .
\end{gathered}
$$

Thus, for each $k$ and such a $\beta$ fixed, by Theorem 8 , we have a functional

$$
a_{k, \beta}(x), \quad x \in X_{\beta},
$$

whose critical points give rise to solutions of $(\mathrm{HS})_{k}$. Similarly we have a functional

$$
a_{\gamma, \beta}(x), \quad x \in X_{\beta},
$$

whose critical points give rise to solutions of the following systems (HS) ${ }_{\gamma}$

$$
\begin{gathered}
\tilde{F} z=\gamma z, \\
z(t) \longrightarrow 0, \quad z^{\prime}(t) \longrightarrow 0, \quad t \longrightarrow \infty .
\end{gathered}
$$

For notational simplicity, we denote $a_{k}, a_{\gamma}$ for $a_{k, \beta}$ and $a_{\gamma, \beta}$. Define

$$
\Phi_{k}(z)=\int_{-\infty}^{\infty} R_{k}(t, z) d t
$$

For the functional $a_{k}$, similar to Lemma 15, we have the following lemma.

Lemma 17. (1) $a_{k}$ satisfies (PS) condition, and the critical point set of $a_{k}$ is compact;

(2) $H_{q}\left(X_{\beta},\left(a_{k}\right)_{\alpha_{k}} ; \mathbb{R}\right) \cong \delta_{q, r_{\beta}} \mathbb{R}, q=0,1, \ldots$ for $-\alpha_{k} \in \mathbb{R}$ large enough, where $r_{\beta}=\operatorname{dim}\left(E^{-}\left(X_{\beta}\right)\right)+\mu_{F}\left(K \gamma I_{2 N}\right)$.

Proof. The proof is similar to Lemma 15. From Theorem 11, we have

$$
\begin{aligned}
\left\|a_{k}^{\prime}(x)-a_{\gamma}^{\prime \prime}(0) x\right\|_{L^{2}} & =\left\|P_{\beta} K\left(\nabla_{z} \Phi_{k}\left(z_{k}(x)\right)-\gamma x\right)\right\|_{L^{2}} \\
& \leq\left\|P_{\beta}\left(\nabla_{z} R_{k}\left(z_{k}(x)\right)-\gamma z_{k}(x)\right)\right\|_{L^{2}} \\
& \leq\left\|\left(\nabla_{z} R_{k}\left(z_{k}(x)\right)-\gamma z_{k}(x)\right)\right\|_{L^{2}} .
\end{aligned}
$$


Similar to $(76)$, for $b \in(0,1)$, there is some $c>0$, such that

$$
\left|\nabla_{z} R_{k}\left(t, z_{k}\right)-\gamma z_{k}\right| \leq c\left|z_{k}(t)\right|, \quad \forall t \in \mathbb{R} .
$$

Choose $b \in((1-\alpha) /(1+\alpha), 1)$, similar to (77), and we have

$$
\left\|\nabla_{z} R_{k}\left(t, z_{k}\right)-\gamma z_{k}\right\|_{L^{2}}^{2} \leq c\left\|z_{k}\right\|_{L^{1+b}}^{1+b} .
$$

From Lemma 1, Remark 10, and (91),

$$
\begin{aligned}
\left\|\nabla_{z} R_{k}\left(t, z_{k}\right)-\gamma z_{k}\right\|_{L^{2}}^{2} & \leq c\left\|z_{k}\right\|_{E}^{1+b} \\
& \leq c(\beta)\|x\|_{L^{2}}^{1+b} .
\end{aligned}
$$

From (89) and (92), we have

$$
\left\|a_{k}^{\prime}(x)-a_{\gamma}^{\prime \prime}(0) x\right\|_{L^{2}}^{2} \leq c(\beta)\|x\|_{L^{2}}^{1+b} .
$$

Now, for each $k \in \mathbb{N}$, we assume that $\left\{x_{m}\right\} \subset X_{\beta}$ satisfying $\left\|a_{k}^{\prime}\left(x_{m}\right)\right\| \rightarrow 0$. By $\nu_{F}\left(K \gamma I_{2 N}\right)=0$, we have that $a_{\gamma}^{\prime \prime}(0)$ is invertible on $X_{\beta}$, since $b<1$ the sequence $\left\{x_{m}\right\}$ must be bounded. Thus the (PS) condition for $a_{k}$ holds. From the same reason, we have the compactness of the critical point set of $a_{k}$. And by Lemma 5.1 in Chapter II of [21], we have

$$
H_{q}\left(X_{\beta},\left(a_{k}\right)_{\alpha_{k}} ; \mathbb{R}\right) \cong \delta_{q, r_{\beta}} \mathbb{R}, \quad q=0,1, \ldots,
$$

for $-\alpha_{k} \in \mathbb{R}$ large enough.

Lemma 18. There exist $c>0$, such that for any $k \in \mathbb{N}$, and $z \in$ $L^{2}$ satisfies the systems $(H S)_{k}$, if $\mu_{F}(z) \leq \mu_{F}\left(K B_{\infty}\right)-1$, one has $\|z\|_{L^{\infty}} \leq c$.

Proof. We prove it indirectly. Assume there exist $R_{k}$ and $z_{k}$ satisfying the conditions, and $\left\|z_{k}\right\|_{L^{\infty}} \rightarrow \infty$; that is, $\|z\|_{\widetilde{F}} \rightarrow$ $\infty$. Since $\left|\nabla_{z} R_{k}(t, z)\right|<c|z|$, for all $t \in \mathbb{R}, z \in \mathbb{R}^{2 N}$, we have $\left\|z_{k}\right\|_{\widetilde{F}} \leq c\left\|z_{k}\right\|_{L^{2}}$. Denote that $y_{k}=z_{k} /\left\|z_{k}\right\|_{\widetilde{F}}$; then, we have $y_{k} \rightarrow y$ in $L^{2}$ for some $y \in L^{2}$ with $\|y\|_{L^{2}}>0$, and

$$
\widetilde{F} y_{k}=\frac{R_{k}^{\prime}\left(t, z_{k}\right)}{\left\|z_{k}\right\|_{\widetilde{F}}} .
$$

Then, for any $r>0$, there exists $C_{r}>0$, satisfying

$$
\left|\dot{y}_{k}(t)\right| \leq C_{r}\left|y_{k}(t)\right|, \quad t \in I_{r}
$$

where $I_{r}=[-r, r]$. Since $\|y\|_{L^{2}}>0$, there is a $r_{0}>0$, such that

$$
\|y\|_{L^{2}(-r, r)}>\frac{1}{2}\|y\|_{L^{2}}>0, \quad \forall r>r_{0} .
$$

Then, from the similar argument in [23], there is a subsequence throught which we may assume that $\left\{y_{k}\right\}$ converges in uniform norm to $y$, and $y(t) \neq 0$, for all $t \in I_{r}$. Therefore $\left|z_{k}(t)\right| \rightarrow \infty$ uniformly on $I_{r}$, and there is $K(r)$ depending on $r$, such that $\left|z_{k}(t)\right| \geq R_{0}$, for any $t \in I_{r}$ and $k \geq K(r)$.

Performing the saddle point reduction on the following systems:

$$
\begin{gathered}
\widetilde{F} z=B_{\infty} z, \\
z(t) \longrightarrow 0, \quad z^{\prime}(t) \longrightarrow 0, \quad t \longrightarrow \infty,
\end{gathered}
$$

for $\beta$ large enough, we have the functional $a_{\infty, \beta}$ (denote by $a_{\infty}$ for simplicity) and the function $z(x)$; since $v_{F}\left(K B_{\infty}\right)=0$, we have the following decomposition:

$$
X_{\beta}=X_{\beta}^{+}+X_{\beta}^{-}
$$

where $a_{\infty}^{\prime \prime}(0)$ is positive definite on $X_{\beta}^{+}$and negative definite on $X_{\beta}^{-}$. From Remark 10 , and $v_{F}\left(K B_{\infty}\right)=0$, there exists $\alpha>0$, such that for $\beta$ large enough,

$$
\left(\left(\widetilde{F}-B_{\infty}(t)\right) x, x\right)_{L^{2}} \leq-\alpha\|x\|_{L^{2}}^{2}, \quad \forall x \in X_{\beta}^{-} .
$$

From the uniform boundary of $\nabla_{z}^{2} R_{k}(t, z)$ and Remark 10, we can choose $\beta$ large enough, such that

$$
\|\left(\nabla_{z}^{2} R_{k}\left(t, z_{k}\right) z_{k}^{\prime \pm}\left(x_{k}\right) x, x\left\|_{L^{2}} \leq \frac{\alpha}{4}\right\| x \|_{L^{2}}, \quad \forall x \in L^{2},\right.
$$

where $x_{k}=P_{\beta} z_{k}, z_{k}\left(x_{k}\right)=z_{k}(t)$ defined in Theorem 8 . Choose $\varepsilon>0$ small enough and $\varepsilon<\alpha / 4$, such that $\mu_{F}\left(K B_{\infty}\right)=\mu_{F}\left(K\left(B_{\infty}-\varepsilon \cdot I d\right)\right)$. Since $X_{\beta}^{-}$is finite dimensional space, choose $r$ large enough, such that

$$
\left(\left(\nabla_{z}^{2} R_{k}\left(t, z_{k}\right)-B_{\infty}\right) x, x\right)_{L^{2}\left(I_{r}^{c}\right)} \geq-\varepsilon(x, x)_{L^{2}}, \quad \forall x \in X_{\beta}^{-},
$$

where $I_{r}^{c}=\mathbb{R} \backslash I_{r}$, and from the definition of $R_{k}$,

$$
\nabla_{z}^{2} R_{k}\left(t, z_{k}(t)\right) \geq B_{\infty}(t), \quad t \in I_{r}, k \geq K(r)
$$

that is,

$$
\left(\left(\nabla_{z}^{2} R_{k}\left(t, z_{k}\right)-B_{\infty}\right) x, x\right)_{L^{2}\left(I_{r}\right)} \geq 0, \quad \forall x \in X_{\beta}^{-}, k \geq K(r) .
$$

From (101) and (103),

$$
\left(\nabla_{z}^{2} R_{k}\left(t, z_{k}\right) x, x\right)_{L^{2}} \geq\left(B_{\infty} x, x\right)_{L^{2}}-\varepsilon(x, x)_{L^{2}},
$$

for $k$ large enough. Thus we have

$$
\begin{aligned}
\left(a_{k}^{\prime \prime}\right. & \left.\left(x_{k}\right) x, x\right)_{L^{2}} \\
= & \left(\left(\widetilde{F}-\nabla_{z}^{2} R_{k}\left(t, z_{k}\right)\right) x, x\right)_{L^{2}} \\
& -\left(\nabla_{z}^{2}\left(R_{k}\left(t, z_{k}\right)\right)\left(z_{k}^{\prime+}+z_{k}^{\prime-}\right) x, x\right)_{L^{2}} \\
\leq & \left(\left(\widetilde{F}-B_{\infty}\right) x, x\right)_{L^{2}}+\frac{\alpha}{2}\|x\|_{L^{2}}^{2}+\varepsilon\|x\|_{L^{2}}^{2} \\
\leq & -\frac{\alpha}{4}\|x\|_{L^{2}}^{2} .
\end{aligned}
$$

That is, $m_{a_{k}}^{-}(x) \geq m_{a_{\infty}}^{-}(0)$, from Theorem 11, $m_{a_{k}}^{-}\left(x_{k}\right)=$ $\operatorname{dim}\left(E^{-}\left(\mathscr{H}_{0}\right)\right)+\mu_{F}\left(z_{x}\left(x_{k}\right)\right), m_{a_{\infty}}^{-}(0)=\operatorname{dim}\left(E^{-}\left(\mathscr{H}_{0}\right)\right)+$ $\mu_{F}\left(K B_{\infty}\right)$, and thus $\mu_{F}\left(z_{x}\right) \geq \mu_{F}\left(K B_{\infty}\right)$, which contradicts the assumption. 
Proof of Theorem 3. As claimed in Remark 13, we can only consider the case of $\left(R_{\infty}^{+}\right)$. Note that $z=0$ is a critical point of $a_{k}$, and the morse index of 0 for $a_{k}$ is $m_{a_{k}}^{-}(0)=\operatorname{dim}\left(E^{-}\left(\mathscr{H}_{0}\right)\right)+$ $\mu_{F}\left(K B_{0}\right)$, since $\gamma \cdot I_{2 N}>B_{\infty}$, we have

$$
\mu_{F}\left(K \gamma \cdot I_{2 N}\right) \geq \mu_{F}\left(K B_{\infty}\right) \geq \mu_{F}\left(K B_{0}\right) .
$$

From proposition (2) in Lemma 17, use the $\left(m_{a_{k}}^{-}(0)\right)$ th and $\left(m_{a_{k}}^{-}(0)+1\right)$ th Morse inequalities, and $a_{k}$ has a nontrivial critical point $x_{k}$ with its morse index $m_{a_{k}}^{-}\left(x_{k}\right) \leq m_{a_{k}}^{-}(0)+1$; that is, $\mu_{F}\left(z_{k}\right) \leq \mu_{F}\left(K B_{0}\right)+1 \leq \mu_{F}\left(K B_{\infty}\right)-1$, then from Lemma 18, we have that $\left\{z_{k}\right\}$ is bounded in $L^{\infty}$. Thus $z_{k}$ is a nontrivial solution of (HS) for $k$ large enough.

The proof of Theorem 4 is similar to the proof of Theorem 3. Instead of Morse theory we make use of minimax arguments for multiplicity of critical points.

Let $X$ be a Hilbert space and assume $\phi \in C^{2}(X, \mathbb{R})$ is an even functional, satisfying the (PS) condition and $\phi(0)=0$. Denote $S_{c}=\{u \in \mid X\|u\|=c\}$.

Lemma 19 (see [30, Corollary 10.19]). Assume that $Y$ and $Z$ are subspaces of $X$ satisfying $\operatorname{dim} Y=j>k=\operatorname{codim} Z$. If there exist $R>r>0$ and $\alpha>0$ such that

$$
\inf \phi\left(S_{r} \cap Z\right) \geq \alpha, \quad \sup \phi\left(S_{R} \cap Y\right) \leq 0
$$

then $\phi$ has $j-k$ pairs of nontrivial critical points $\left\{ \pm x_{1}, \pm x_{2}, \ldots\right.$, $\left.\pm x_{j-k}\right\}$, so that $\mu\left(x_{i}\right) \leq k+i$, for $i=1,2, \ldots, j-k$.

First, we consider the case of $\left(R_{\infty}^{+}\right)$, since $R$ is even, we have that $R_{k}$ is also even and satisfies Lemma 16 . Let $Y=X_{\beta}^{-}$, and $Z$ the positive space of $a_{k}^{\prime \prime}(0)$ in $X_{\beta}$, and we have and $\operatorname{dim} Y=E^{-}\left(X_{\beta}\right)+\mu_{F}\left(K B_{\infty}\right), \operatorname{codim} Z=E^{-}\left(X_{\beta}\right)+\mu_{F}\left(K B_{0}\right)$, $\operatorname{dim} Y>\operatorname{codim} Z$. So, $a_{k}$ has $l:=\mu_{F}\left(K B_{\infty}\right)-\mu_{F}\left(K B_{0}\right)$ pairs of nontrivial critical points

$$
\left\{ \pm x_{1}, \pm x_{2}, \ldots, \pm x_{l}\right\}
$$

and $l-1$ pairs of them satisfy

$$
m^{-}\left(x_{i}\right) \leq \mu_{F}\left(K B_{0}\right)+i<\mu_{F}\left(K B_{\infty}\right), \quad i=1,2, \ldots, l-1 .
$$

Then, we can complete the proof. In order to prove the case of $\left(R_{\infty}^{-}\right)$, we need the following lemma.

Lemma 20 (see [21, Corollary II 4.1]). Assume that $Y$ and $Z$ are subspaces of $X$ satisfying $\operatorname{dim} Y=j>k=\operatorname{codim} Z$. If there exist $r>0$, and $\alpha>0$ such that

$$
\inf \phi(Z)>-\infty, \quad \sup \phi\left(S_{r} \cap Y\right) \leq-\alpha,
$$

then $\phi$ has $j-k$ pairs of nontrivial critical points $\pm u_{1}, \pm u_{2}, \ldots$, $\pm u_{j-k}$ so that $\mu\left(u_{i}\right)+v\left(u_{i}\right) \geq k+i-1$ for $i=1,2, \ldots, j-k$. here.

The proof is similar to the case of $\left(R_{\infty}^{+}\right)$, and we omit it

\section{Acknowledgment}

This work is partially supported by NFSC $(11126154,11126146$, and 11201196).

\section{References}

[1] G. Arioli and A. Szulkin, "Homoclinic solutions of Hamiltonian systems with symmetry," Journal of Differential Equations, vol. 158, no. 2, pp. 291-313, 1999.

[2] G. Chen and S. Ma, "Homoclinic orbits of superlinear Hamiltonian systems," Proceedings of the American Mathematical Society, vol. 139, no. 11, pp. 3973-3983, 2011.

[3] V. C. Zelati, I. Ekeland, and E. Séré, "A variational approach to homoclinic orbits in Hamiltonian systems," Mathematische Annalen, vol. 288, no. 1, pp. 133-160, 1990.

[4] Y. Ding, "Multiple homoclinics in a Hamiltonian system with asymptotically or super linear terms," Communications in Contemporary Mathematics, vol. 8, no. 4, pp. 453-480, 2006.

[5] Y. Ding and M. Girardi, "Infinitely many homoclinic orbits of a Hamiltonian system with symmetry," Nonlinear Analysis: Theory, Methods \& Applications, vol. 38, no. 3, pp. 391-415, 1999.

[6] Y. Ding and M. Willem, "Homoclinic orbits of a Hamiltonian system," Zeitschrift für Angewandte Mathematik und Physik, vol. 50, no. 5, pp. 759-778, 1999.

[7] H. Hofer and K. Wysocki, "First order elliptic systems and the existence of homoclinic orbits in Hamiltonian systems," Mathematische Annalen, vol. 288, no. 3, pp. 483-503, 1990.

[8] E. Séré, "Looking for the Bernoulli shift," Annales de l'Institut Henri Poincaré. Analyse Non Linéaire, vol. 10, no. 5, pp. 561-590, 1993.

[9] E. Séré, "Existence of infinitely many homoclinic orbits in Hamiltonian systems," Mathematische Zeitschrift, vol. 209, no. 1, pp. 27-42, 1992.

[10] J. Sun, J. Chu, and Z. Feng, "Homoclinic orbits for first order periodic Hamiltonian systems with spectrum point zero," Discrete and Continuous Dynamical Systems, vol. 33, no. 8, pp. 12411257, 2013.

[11] A. Szulkin and W. Zou, "Homoclinic orbits for asymptotically linear Hamiltonian systems," Journal of Functional Analysis, vol. 187, no. 1, pp. 25-41, 2001.

[12] K. Tanaka, "Homoclinic orbits in a first order superquadratic Hamiltonian system: convergence of subharmonic orbits," Journal of Differential Equations, vol. 94, no. 2, pp. 315-339, 1991.

[13] Y. H. Ding and S. J. Li, "Homoclinic orbits for first order Hamiltonian systems," Journal of Mathematical Analysis and Applications, vol. 189, no. 2, pp. 585-601, 1995.

[14] Y. Ding and L. Jeanjean, "Homoclinic orbits for a nonperiodic Hamiltonian system," Journal of Differential Equations, vol. 237, no. 2, pp. 473-490, 2007.

[15] Y. Ding and C. Lee, "Existence and exponential decay of homoclinics in a nonperiodic superquadratic Hamiltonian system," Journal of Differential Equations, vol. 246, no. 7, pp. 2829-2848, 2009.

[16] W. Qin, J. Zhang, and F. Zhao, "Homoclinic orbits for a class of nonperiodic Hamiltonian systems," Abstract and Applied Analysis, vol. 2012, Article ID 769232, 20 pages, 2012.

[17] J. Sun, H. Chen, and J. J. Nieto, "Homoclinic orbits for a class of first-order nonperiodic asymptotically quadratic Hamiltonian systems with spectrum point zero," Journal of Mathematical Analysis and Applications, vol. 378, no. 1, pp. 117-127, 2011. 
[18] C. N. Chen and X. Hu, "Maslov index for homoclinic orbits of Hamiltonian systems," Annales de l'Institut Henri Poincaré. Analyse Non Linéaire, vol. 24, no. 4, pp. 589-603, 2007.

[19] C. Zhu and Y. Long, "Maslov-type index theory for symplectic paths and spectral flow. I," Chinese Annals of Mathematics B, vol. 20, no. 4, pp. 413-424, 1999.

[20] H. Amann, "Saddle points and multiple solutions of differential equations," Mathematische Zeitschrift, vol. 169, no. 2, pp. 127$166,1979$.

[21] K. C. Chang, Infinite-Dimensional Morse Theory and Multiple Solution Problems, Progress in Nonlinear Differential Equations and their Applications, Birkhäauser, Basel, Switzerland, 1993.

[22] Y. Long, Index Theory for Symplectic Paths with Applications, vol. 207 of Progress in Mathematics, Birkhäuser, Basel, Switzerland, 2002.

[23] Z. Liu, J. Su, and Z. Q. Wang, "A twist condition and periodic solutions of Hamiltonian systems," Advances in Mathematics, vol. 218, no. 6, pp. 1895-1913, 2008.

[24] M. F. Atiyah, V. K. Patodi, and I. M. Singer, "Spectral asymmetry and Riemannian geometry. III," Mathematical Proceedings of the Cambridge Philosophical Society, vol. 79, no. 1, pp. 71-99, 1976.

[25] S. E. Cappell, R. Lee, and E. Y. Miller, "On the Maslov index," Communications on Pure and Applied Mathematics, vol. 47, no. 2, pp. 121-186, 1994.

[26] A. Floer, "A relative Morse index for the symplectic action," Communications on Pure and Applied Mathematics, vol. 41, no. 4, pp. 393-407, 1988.

[27] J. Robbin and D. Salamon, “The Maslov index for paths," Topology, vol. 32, no. 4, pp. 827-844, 1993.

[28] J. Robbin and D. Salamon, "The spectral flow and the Maslov index," The Bulletin of the London Mathematical Society, vol. 27, no. 1, pp. 1-33, 1995.

[29] Z. Liu, J. Su, and Z. Q. Wang, "Solutions of elliptic problems with nonlinearities of linear growth," Calculus of Variations and Partial Differential Equations, vol. 35, no. 4, pp. 463-480, 2009.

[30] N. Ghoussoub, Duality and Perturbation Methods in Critical Point Theory, vol. 107 of Cambridge Tracts in Mathematics, Cambridge University Press, Cambridge, UK, 1993. 


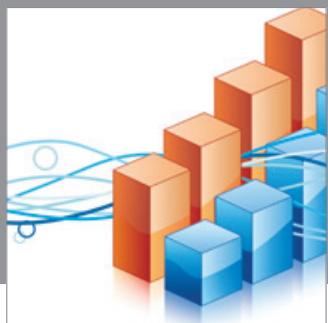

Advances in

Operations Research

mansans

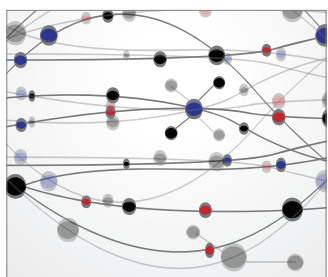

The Scientific World Journal
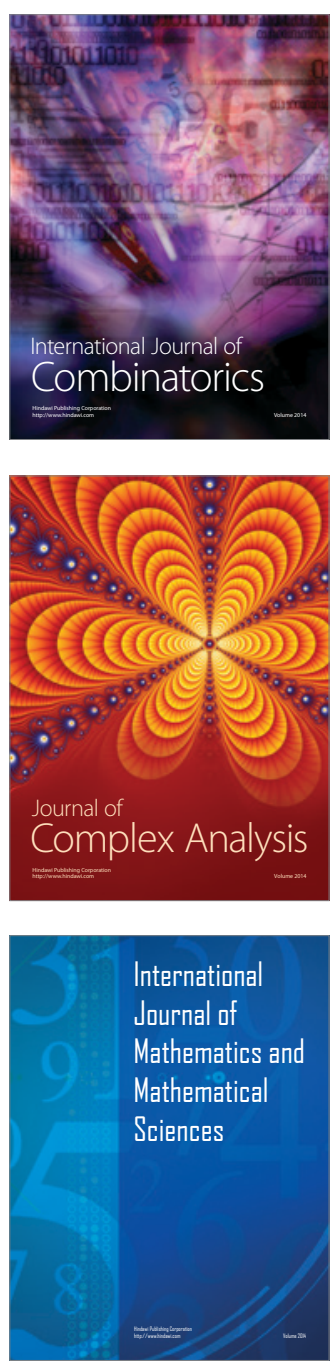
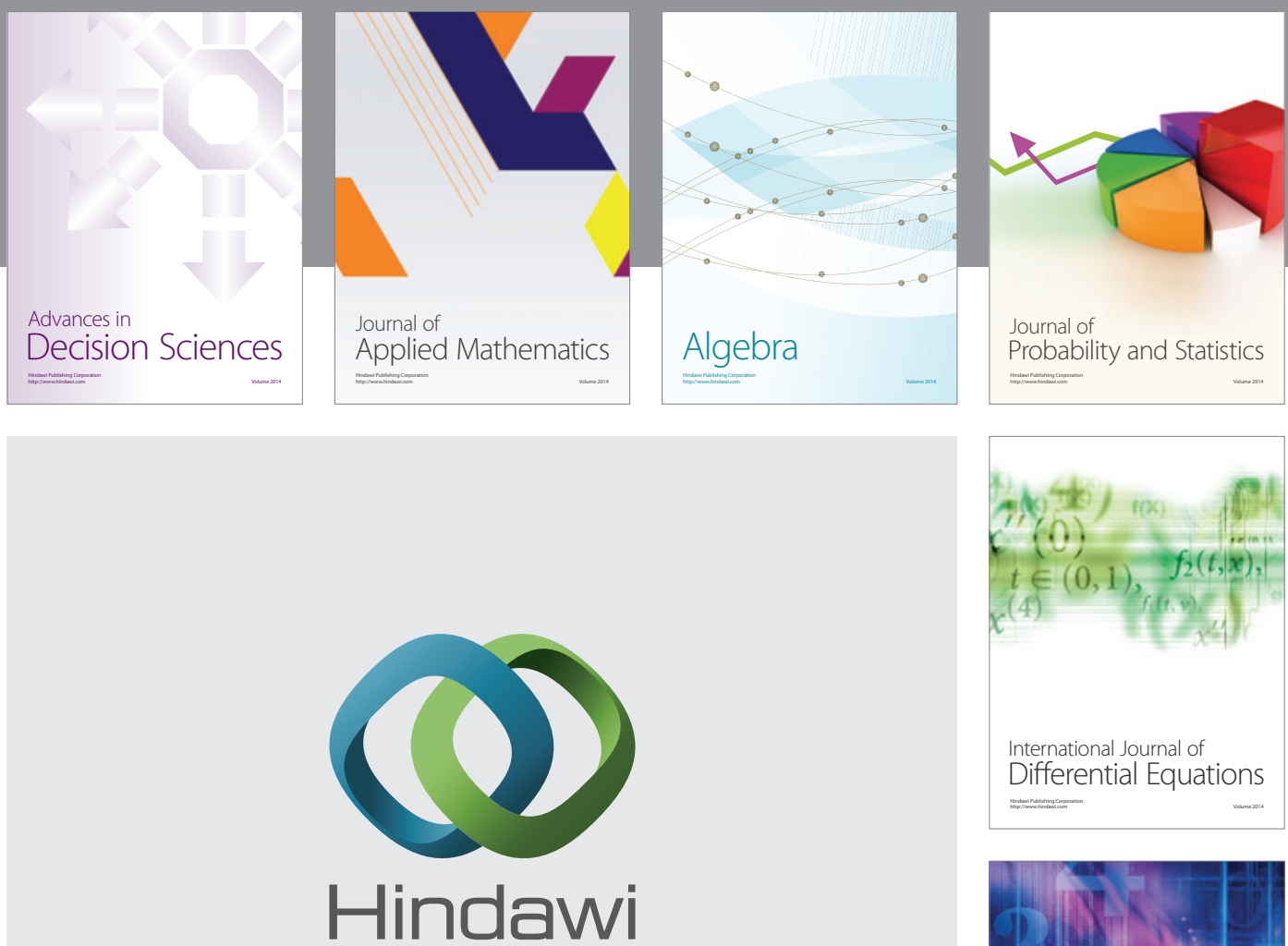

Submit your manuscripts at http://www.hindawi.com
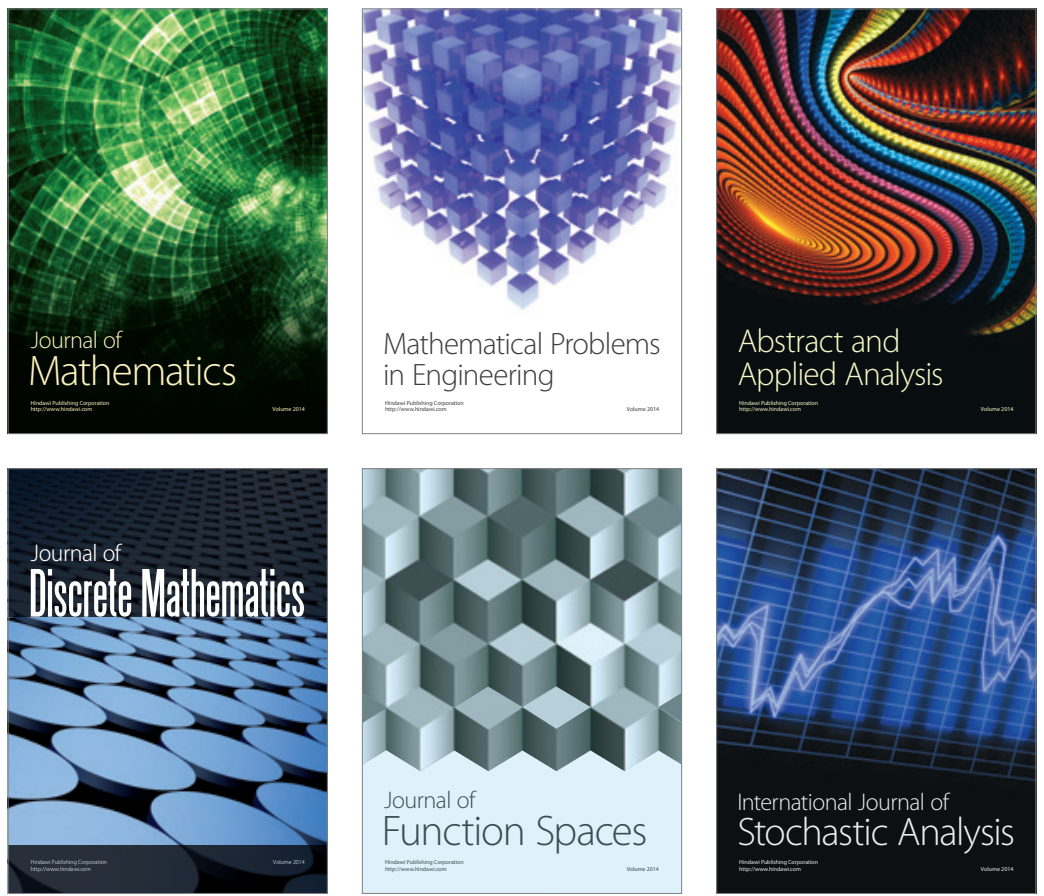

Journal of

Function Spaces

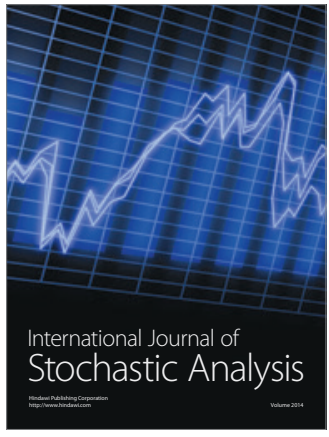

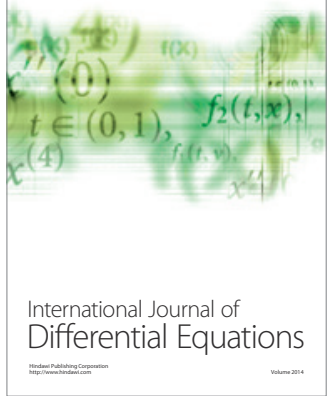
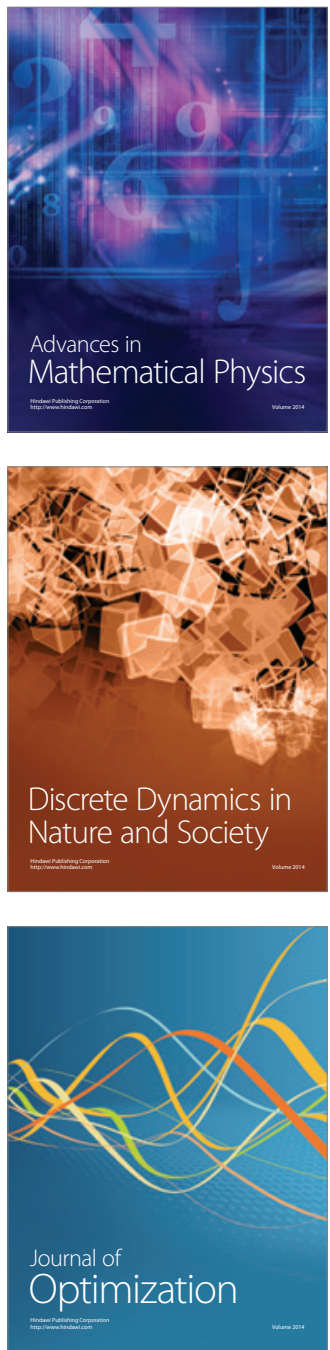